\title{
Persistent Quasi-periodic Pulsations during a Large X-class Solar Flare
}

\section{Hayes, Laura A.}

2019-04-10

Hayes , L A , Gallagher , P T , Dennis , B R , Ireland , J , Inglis , A \& Morosan , D E 2019 , ' Persistent Quasi-periodic Pulsations during a Large X-class Solar Flare ' , Astrophysical Journal , vol. 875 , no. 1 , 33 . https://doi.org/10.3847/1538-4357/ab0ca3

http://hdl.handle.net/10138/303515

https://doi.org/10.3847/1538-4357/ab0ca3

unspecified

publishedVersion

Downloaded from Helda, University of Helsinki institutional repository.

This is an electronic reprint of the original article.

This reprint may differ from the original in pagination and typographic detail.

Please cite the original version. 


\title{
Persistent Quasi-periodic Pulsations during a Large X-class Solar Flare
}

\author{
Laura A. Hayes ${ }^{1,2}$ (1) , Peter T. Gallagher ${ }_{1}^{1,3}$, Brian R. Dennis ${ }^{2}$ (1) Jack Ireland $^{2}$ (1) , Andrew Inglis ${ }^{2}$ (1), and Diana E. Morosan ${ }^{4}$ \\ ${ }^{1}$ School of Physics, Trinity College Dublin, Dublin 2, Ireland \\ ${ }^{2}$ Solar Physics Laboratory, Code 671, Heliophysics Science Division, NASA Goddard Space Flight Center, Greenbelt, MD 20771, USA \\ ${ }^{3}$ School of Cosmic Physics, Dublin Institute for Advanced Studies, Dublin, D02XF85, Ireland \\ ${ }^{4}$ Department of Physics, University of Helsinki, P.0. Box 64, Helsinki, Finland \\ Received 2018 December 20; revised 2019 March 2; accepted 2019 March 4; published 2019 April 10
}

\begin{abstract}
Solar flares often display pulsating and oscillatory signatures in the emission, known as quasi-periodic pulsations (QPP). QPP are typically identified during the impulsive phase of flares, yet in some cases, their presence is detected late into the decay phase. Here, we report extensive fine structure QPP that are detected throughout the large X8.2 flare from 2017 September 10. Following the analysis of the thermal pulsations observed in the Geostationary Operational Environmental Satellite/X-ray sensor and the $131 \AA$ channel of Solar Dynamics Observatory/Atmospheric Imaging Assembly, we find a pulsation period of $\sim 65 \mathrm{~s}$ during the impulsive phase followed by lower amplitude QPP with a period of $\sim 150 \mathrm{~s}$ in the decay phase, up to three hours after the peak of the flare. We find that during the time of the impulsive QPP, the soft X-ray source observed with the Reuven Ramaty High Energy Solar Spectroscopic Imager rapidly rises at a velocity of approximately $17 \mathrm{~km} \mathrm{~s}^{-1}$ following the plasmoid/coronal mass ejection eruption. We interpret these QPP in terms of a manifestation of the reconnection dynamics in the eruptive event. During the long-duration decay phase lasting several hours, extended downward contractions of collapsing loops/plasmoids that reach the top of the flare arcade are observed in EUV. We note that the existence of persistent QPP into the decay phase of this flare are most likely related to these features. The QPP during this phase are discussed in terms of magnetohydrodynamic wave modes triggered in the post-flaring loops.
\end{abstract}

Key words: Sun: flares - Sun: oscillations - Sun: X-rays, gamma rays

Supporting material: animation

\section{Introduction}

A common characteristic in solar and stellar flaring emission is the presence of oscillatory or pulsating signatures known as quasi-periodic pulsations (QPP). QPP have typical periods ranging from seconds to several minutes, and their presence in both a solar and stellar context has been widely discussed in the literature (see Nakariakov \& Melnikov 2009 and Van Doorsselaere et al. 2016 for recent comprehensive reviews).

QPP are prominently identified during the impulsive phase of flares in nonthermal hard X-ray and microwave observations (Parks \& Winckler 1969; Fleishman et al. 2008; Inglis \& Dennis 2012; Hayes et al. 2016; Huang et al. 2016). However, recent studies have provided evidence of QPP signatures across the whole electromagnetic spectrum of flaring emissions-e.g., $\gamma$-rays (Nakariakov et al. 2010), Ly $\alpha$ (Milligan et al. 2017), chromospheric line emission (Brosius et al. 2016) and Doppler shift velocities (Tian et al. 2016), soft X-ray and EUV (Dolla et al. 2012; Simões et al. 2015; Dennis et al. 2017; Dominique et al. 2018), and decimetric radio bursts ( $\mathrm{Li}$ et al. 2015; Kupriyanova et al. 2016). Given that QPP are directly linked to all aspects of flaring energy release, their observations provide clues to the physical processes operating in the flare site.

Despite the increased number of observations, the nature and underlying physical mechanism for the generation of QPP remain debated. Various mechanisms have been suggested regarding possible causes of QPP in solar and stellar flares (see McLaughlin et al. 2018 for a recent review of QPP models). These mechanisms can be categorized into either oscillatory or self-oscillatory processes.
In the oscillatory category, QPP are described in terms of motions around an equilibrium - such as magnetohydrodynamic (MHD) oscillations in or near the energy release site. The observed periodicities of QPP are consistent with the expected timescales of MHD waves in coronal conditions, making them an attractive explanation. For example, the decayless regime of kink mode oscillations (e.g., Anfinogentov et al. 2013, 2015), now identified as a common phenomenon in flaring active region, have timescales that overlap with that of flaring QPP and may explain the presence of low-amplitude QPP that exist for several cycles of oscillation. MHD waves propagating in flaring loops could periodically modulate the emission directly or affect charged particle dynamics (Nakariakov \& Melnikov 2009). MHD waves can also play a role in the periodic triggering of magnetic reconnection (Nakariakov et al. 2006; Nakariakov \& Zimovets 2011). The observed QPP period would depend on the properties of the flaring loops and the type of MHD wave mode (e.g., sausage, kink, slow magneto-acoustic). When interpreted in terms of MHD wave modes, QPP observations offer an opportunity to perform coronal seismology - the inference of magnetic and plasma properties from observed oscillatory behavior in the corona (Nakariakov \& Melnikov 2009; De Moortel \& Nakariakov 2012).

In the self-oscillatory case, the QPP are connected to the dynamics of magnetic reconnection and energy release, which may be time-dependent. Numerical MHD simulations have shown that reconnection does not proceed in a steady fashion, but instead exhibits a time-dependent behavior, producing periodic outputs from an aperiodic driver (Murray et al. 2009; McLaughlin et al. 2012; Thurgood et al. 2017). Similarly, the 
magnetic island reconnection system (Drake et al. 2006; Guidoni et al. 2016) induces a repetitive regime of reconnection resulting in a periodic acceleration of electrons into flaring loops. The QPP signatures in flaring light curves are then a manifestation of periodic energy release, and their period is related to the timescale of oscillatory reconnection or the generation and interaction of magnetic islands in current sheets.

To date, observations have not allowed for a definitive choice between possible mechanisms. It is quite likely that different processes operate in different cases and during different phases of the flare. Recent work has shown that thermal soft X-ray QPP are a prominent feature in flaring emissions, clearly identified during the impulsive phase in the detrended or time-derivative light curves (Dolla et al. 2012; Simões et al. 2015; Hayes et al. 2016; Kolotkov et al. 2018). In some cases the pulsations are observed to persist late into the decay phase (Hayes et al. 2016; Dennis et al. 2017). These fine structure QPP must be linked to some aspects of the flaring region and their relationship to other flaring parameters requires investigation.

In this paper we investigate the nature of extensive QPP detected in the thermal emissions from the well-observed X8.2 solar flare on 2017 September 10. By using a combination of high cadence soft X-ray measurements, together with spatially resolved observations using the Atmospheric Imaging Assembly (AIA) on board the Solar Dynamics Observatory (SDO; Lemen et al. 2012) and the Reuven Ramaty High Energy Solar Spectroscopic Imager (RHESSI; Lin et al. 2002), we relate the characteristics of the detected QPP to the length scales of the flaring event.

\section{Observations}

The flare analyzed in this paper is the X8.2 solar flare that occurred on 2017 September 10 from NOAA active region 12673. This was the second largest flare of solar cycle 24 and was accompanied by one of the fastest coronal mass ejections (CMEs) observed to date with a speed of $\sim 3000 \mathrm{~km} \mathrm{~s}^{-1}$ (Gopalswamy et al. 2018; Morosan et al. 2019). The position on the western limb of the Sun provided a unique view of the solar eruptive process, and many recent studies of different aspects of the flare have been discussed in the literature (e.g., Gary et al. 2018; Li et al. 2018; Liu et al. 2018; Omodei et al. 2018; Warren et al. 2018).

The flare started at approximately 15:35 UT, reached a peak in the GOES 1-8 $\AA$ channel at 16:06 UT and did not return to preflare flux levels until the next day. Figures 1(a)-(d) shows the evolution of the event as observed in the $131 \AA$ channel of AIA. The pre-eruptive plasmoid is clearly seen in (a) followed by the flare and cusp-shaped post-flaring loops (b)-(d). The bright linear structure extending outward is presumably related to the reconnecting current sheet following the eruption. This feature has been identified as a current sheet in recent works ( $\mathrm{Li}$ et al. 2018; Warren et al. 2018), and turbulence is noted to explain why it appears wider than theoretically expected (Cheng et al. 2018). Here, we will also refer to this structure as the current sheet. However, it should be noted that the structure observed in EUV may not actually be the region where reconnection itself is taking place, but more likely an emission structure perhaps associated with the reconnection outflow jets.

In this paper we focus on the fine structure of the QPP detected in both channels (1-8 $\AA$ and $0.5-4 \AA$ ) of the Geostationary Operational Environmental Satellite's X-ray sensor (GOES/XRS), the 1-70 А soft X-ray channel from the Extreme Ultraviolet Spectrometer (ESP) which is part of the EUV experiment (Woods et al. 2012) on board SDO, and the $131 \AA$ channel of SDO/AIA.

Both GOES and ESP provide soft X-ray high time cadence observations of $2 \mathrm{~s}$ and $0.25 \mathrm{~s}$, respectively. In order to enhance the signal-to-noise ratio of ESP, the signal is summed to $2 \mathrm{~s}$ to match the GOES data. Figure 1(e) shows the light curves of the GOES and ESP channels. In comparison to nonthermal emission in which QPP are usually clearly evident as large modulation depths, QPP in soft X-rays constitute a small fraction $(\lesssim 1 \%)$ of the total emission in this energy range and are difficult to see in the raw light curves. In order to show the variability more clearly, we detrend the data by subtracting a background calculated using a Savitzky-Golay smoothing filter (Savitzky \& Golay 1964; Press et al. 1986) of degree of 3 and a window size of $200 \mathrm{~s}$. This window size is chosen as it was found to appropriately highlight the pulsations of interest. Similarly other window sizes can be chosen, and the QPP can also be enhanced by taking the numerical derivative (e.g., Simões et al. 2015; Hayes et al. 2016). Figures 1(f) and (g) show the detrended light curves of the soft X-ray GOES and ESP channels, respectively. Here QPP can be clearly identified during both the impulsive and decay phases. The similarity of the GOES and ESP QPP confirms that these pulsations are of solar origin and not an instrumental feature.

It should be noted that both GOES and ESP provide Sun-as-astar observations and so their light curves contain disk-integrated flux. To confirm that the observed long duration QPP signatures are associated with the emission from the flaring region of interest, we make integrated AIA $131 \AA$ light curves over the field of view in Figures 1(a)-(d). To avoid loss of signal from the bleeding of saturated pixels in the images, we make sure to include all the saturated pixels in this field of view (i.e., (a)-(d)). The $131 \AA$ channel of AIA has a response to the hot Fe XXI $(10 \mathrm{MK})$ and Fe XXII (16 MK) lines and hence is sensitive to soft $\mathrm{X}$-ray emitting plasma at a similar temperature. The detrended light curve of the $131 \AA$ channel is shown in Figure 1(h). QPP signatures are also clearly evident, particularly extending late into the decay phase, confirming that these long duration persistent QPP are coming from this flaring region. The AIA $131 \AA$ detrended light curves also display two extra large amplitude pulsations during the impulsive phase at approximately 16:12-16:16 UT, which are not observed in GOES or ESP. These are also observed in the AIA $193 \AA$ channel light curves shown in the Appendix (see Figure 9), confirming that they are real. It may be possible that these are associated with the lower temperature plasma that the $131 \AA$ (0.4 MK) and $193 \AA$ (1.2 MK) channels are sensitive to.

During the impulsive phase, the QPP have a more "bursty" nature and have larger amplitude than in the decay phase. The amplitude of the pulsations in all channels is small, on the order of $\sim 1 \%$ of the overall emission during the impulsive phase and $\sim 0.3 \%$ during the decay phase. What is noteworthy here is that the QPP persist for up to $3 \mathrm{hr}$ after the GOES peak. Furthermore, the long-duration pulsations that extend into the decay phase are observed to have a "beat" signature, with wavepackets of larger amplitude pulsations followed by times of lower amplitude pulsations.

In order to investigate the pulsations further, we break the light curves up into an impulsive phase from 15:50 to 16:15 UT (highlighted by the vertical dashed lines in Figures 1 (e)-(h)) 

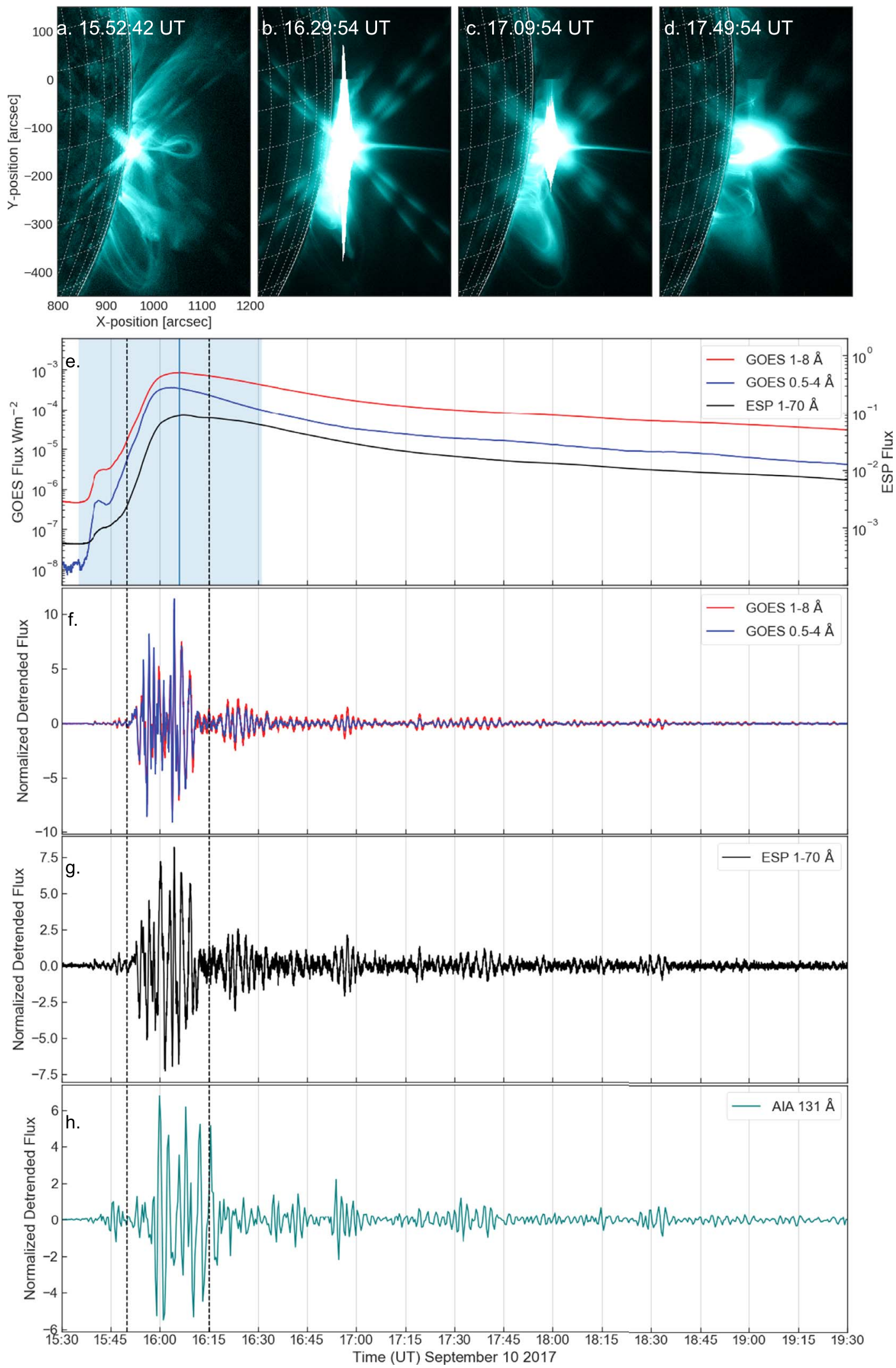

Figure 1. Evolution of the flare seen in EUV (a)-(d) in the AIA 131 A passband. Detector saturation causes the blooming in (b) and (c) given the large magnitude of the event, and the crossed features are the diffraction effects of the detector grids. The soft X-ray light curves from both GOES and ESP are shown in (e). The shaded region denotes the defined start and end times and the blue vertical line is at the peak of GOES 1-8 $\mathrm{A}$ channel. Panels (f), (g), and (h) show the detrended light curves of the two GOES channels, ESP, and AIA $131 \AA$ respectively. The detrended light curves are normalized by dividing by their standard deviation. Persistent QPP can clearly be identified in each channel. The vertical dashed lines in (e)-(h) show the impulsive phase. 

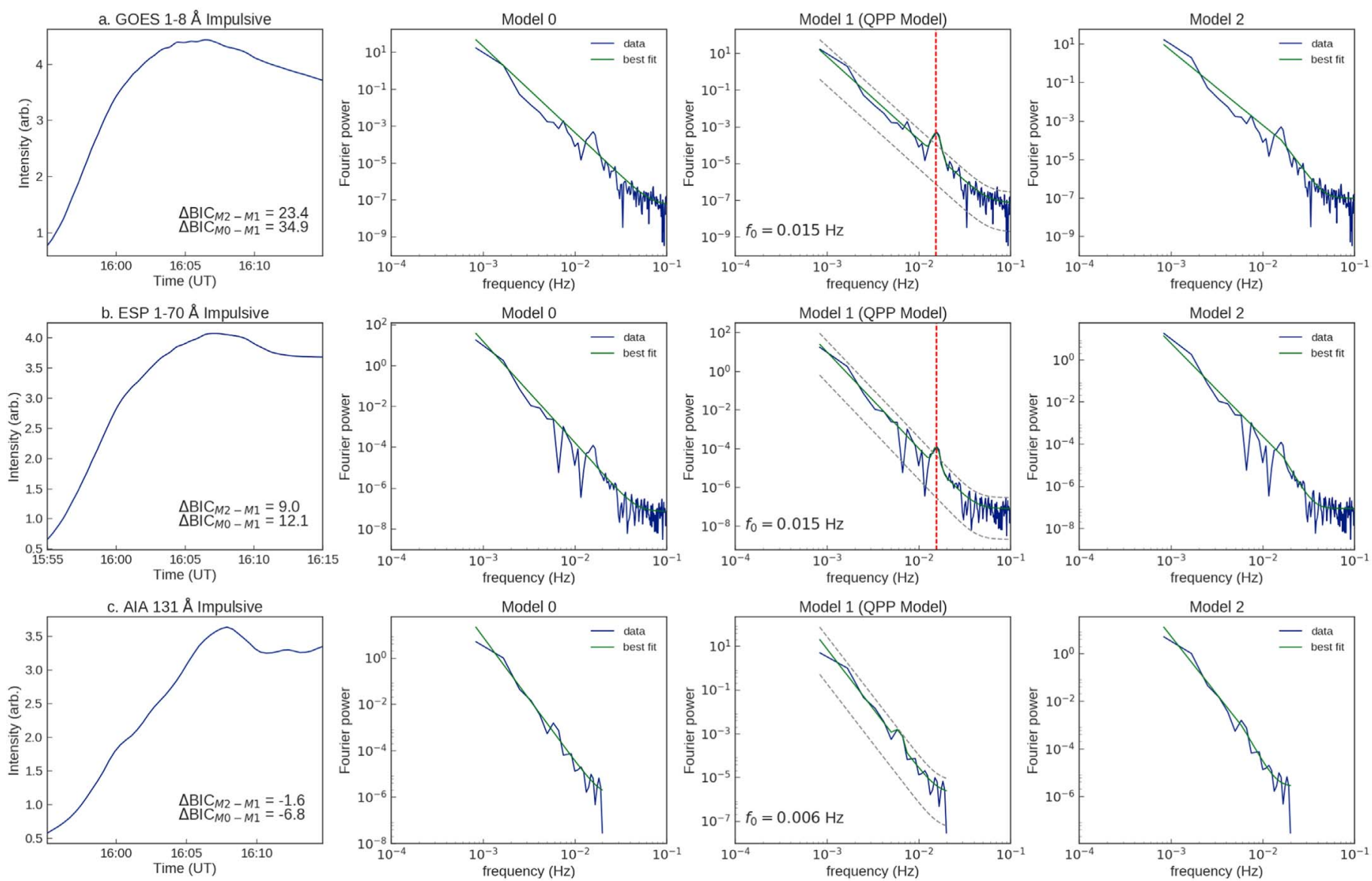

Figure 2. Impulsive phase light curves with AFINO analysis. (a), (b), and (c) correspond to the GOES 1-8 , ESP 1-70 A, and AIA $131 \AA$ A respectively. For each, the original time series is shown in the left panel, and the fits to each model are shown in the remaining panels. If the QPP model is preferred, a red dashed line is shown, as in (a) and (b).

and a decay phase from 16:15 to 19:00 UT. We study the QPP characteristics in these time intervals and their relation to spatial flaring features observed with AIA and RHESSI.

\section{Results}

\subsection{Periodicity}

The presence of QPP is confirmed by periodogram analysis using the Automated Flare Inference of Oscillations (AFINO) method detailed in Inglis et al. $(2015,2016)$. The Fourier power spectra of flaring time series have an intrinsic power-law shape that must be taken into account when assessing the significance of a peak in a periodogram. When a signal is detrended, spectral components are suppressed that can lead to false detections of significant periods (e.g., Gruber et al. 2011; Inglis et al. 2015). The advantage of using AFINO is that the analysis is performed on the raw light curves with no detrending required.

AFINO is outlined in detail in Inglis et al. (2016) but is summarized here. First the raw light curve is normalized by the mean and a Hanning window function is applied to account for the finite duration of the time series. The Fourier power spectrum is then calculated and a model fit and comparison test is performed. There are three models considered to represent the power spectrum-a single power law plus a constant (model 0), a power-law-plus-constant model with a Gaussian bump (model 1), and a broken power-law model (model 2).
Model 1 represents a situation with excess power at a localized frequency-i.e., the QPP model. The maximum likelihood of the three models to fit the power spectra are calculated and then tested against each other to find which model most likely represents the data. The comparison is done via the Bayesian Information Criterion (BIC), see Schwarz (1978) and Inglis et al. $(2015,2016)$ for a detailed explanation. A smaller value of BIC indicates that a model is preferred to others, and hence the $\triangle \mathrm{BIC}$ between model fits represent a way to determine which model is most appropriate. A $\Delta \mathrm{BIC}_{\text {modelA-modelB }}>10$ suggests that model B is strongly preferred over model A (Kass \& Raftery 1995).

AFINO is applied to both the impulsive and decay phases of the light curves separately, and the results are shown in Figures 2 and 3, respectively. Each panel shows the input time series, and the three model fits to the power spectrum. For the QPP model, the $2.5 \%$ and $97.5 \%$ quantiles relative to the power-law component are shown in gray. If the QPP model is preferred, a red dashed line is plotted to denote the peak of the Gaussian bump, $f_{0}$, which is the frequency of the detected QPP oscillation.

It is found that during the impulsive phase, the QPP model is preferred for both the GOES channels and the ESP channel, with peak period of $\sim 65 \mathrm{~s}$ of the enhanced power in both these channels. The impulsive phase of the AIA $131 \AA$ light curve, however, shows no significant enhanced power in the Fourier power spectrum, and the QPP model fit to the spectrum is not 

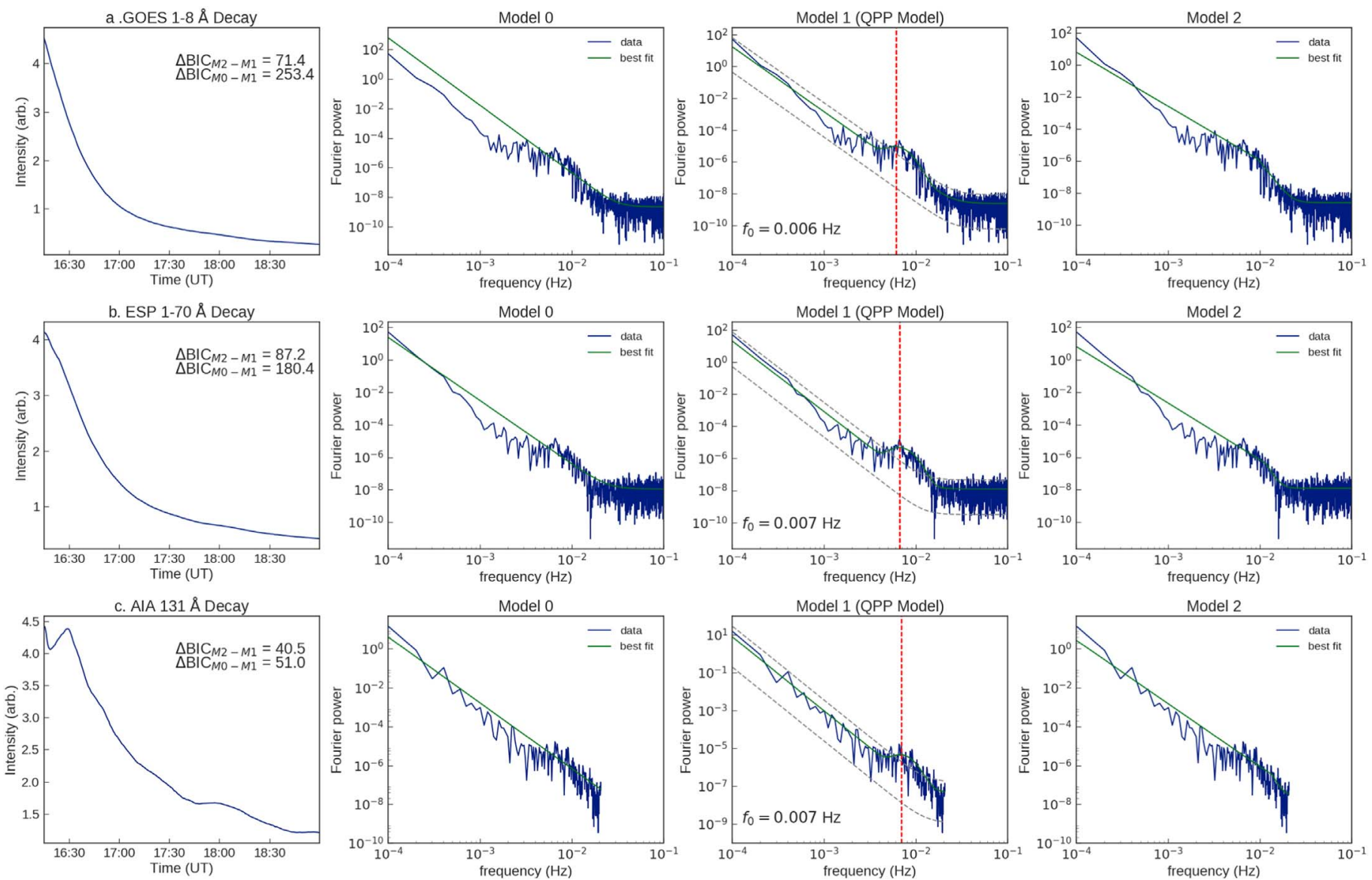

Figure 3. Decay phase light curves with AFINO analysis. The same as Figure 2; however, here all three light curves are best described by the QPP model with a period of $150-160 \mathrm{~s}$.

found to be favored. The time cadence of the AIA $131 \AA$ light curve is $24 \mathrm{~s}$ here to ensure constant exposure time. The period of $65 \mathrm{~s}$ is close to the Nyquist frequency of the sampling of AIA $131 \AA$ and hence makes it difficult to detect if such a period is present. Furthermore, the BIC explicitly penalizes for a small number of data points, and given that there are much less data points for AIA compared to GOES and ESP it makes it difficult to detect a significant period at this time. This does not mean that the pulsations observed in Figure 1(h) are not real during the impulsive phase, just that they are not found to have a significant period with AFINO. The times between the observed peaks are estimated by eye to be $\sim 155 \mathrm{~s}$. However, it again should be noted that perhaps the sampling is not permitting the detection of a shorter period.

In the decay phase, all channels show significant enhanced power in the Fourier power spectrum, and the QPP model is found to fit best for all three instruments. The enhanced power is centered on a period of $\sim 150-160 \mathrm{~s}$. This confirms the visual identification of the long duration, low amplitude QPP seen in the decay phase of Figure 1. It is notable that there is a much longer period ( $150 \mathrm{~s}$ versus $65 \mathrm{~s}$ ) in the decay phase than in the impulsive phase observed in the soft X-ray GOES and ESP channels. Longer timescales during the decay phase have been seen before in Simões et al. (2015), Hayes et al. (2016), and Dennis et al. (2017). This may be attributed to the increase in height of emitting hot plasma in longer loops at the later stages of the flare, or perhaps a different dominant driver of the QPP signatures.

\subsection{X-Ray Sources and Loop Length Estimation}

The magnetic configuration of the solar eruptive event and its position on the limb provides an excellent view of the flare and observations of the post-flare loops face on (see Figures 1(a)-(d)). The flare was well observed by RHESSI, and the on-limb location allows the soft X-ray sources to be imaged and their altitudes determined. A sequence of X-ray images in the $6-12 \mathrm{keV}$ and $12-25 \mathrm{keV}$ energy bands were made using the CLEAN reconstruction algorithm (Hurford et al. 2002) with detectors 3,6 , and 8 , a beamwidth-factor of 1 , and an integration time of $20 \mathrm{~s}$.

The 6-12 keV RHESSI soft X-ray contours overplotted on the AIA $131 \AA$ images are shown in Figure 4. These soft X-ray sources are sources of hot thermal X-ray emission from the flare heated plasma. The soft X-ray sources are located at the top of the loop, presumably at a lower altitude to that of the reconnection region itself. It is observed to rise in altitude as the flare evolves, following the evolution of the EUV loops. To track the height evolution, the centroid value inside the contour of each image in the sequence is determined. The computed height of the centroids above the limb in the plane of the sky is shown in Figure 5. The gaps in the data represent periods when RHESSI is at night and unavailable to make observations. It should be noted that the $12-25 \mathrm{keV}$ source is always above the $6-12 \mathrm{keV}$ source. This spatial split in energy, such that the higher energy source is situated at a higher altitude is consistent with previous works (Gallagher et al. 2002; Liu et al. 2013), and can be attributed to the fact that higher loops are newly 

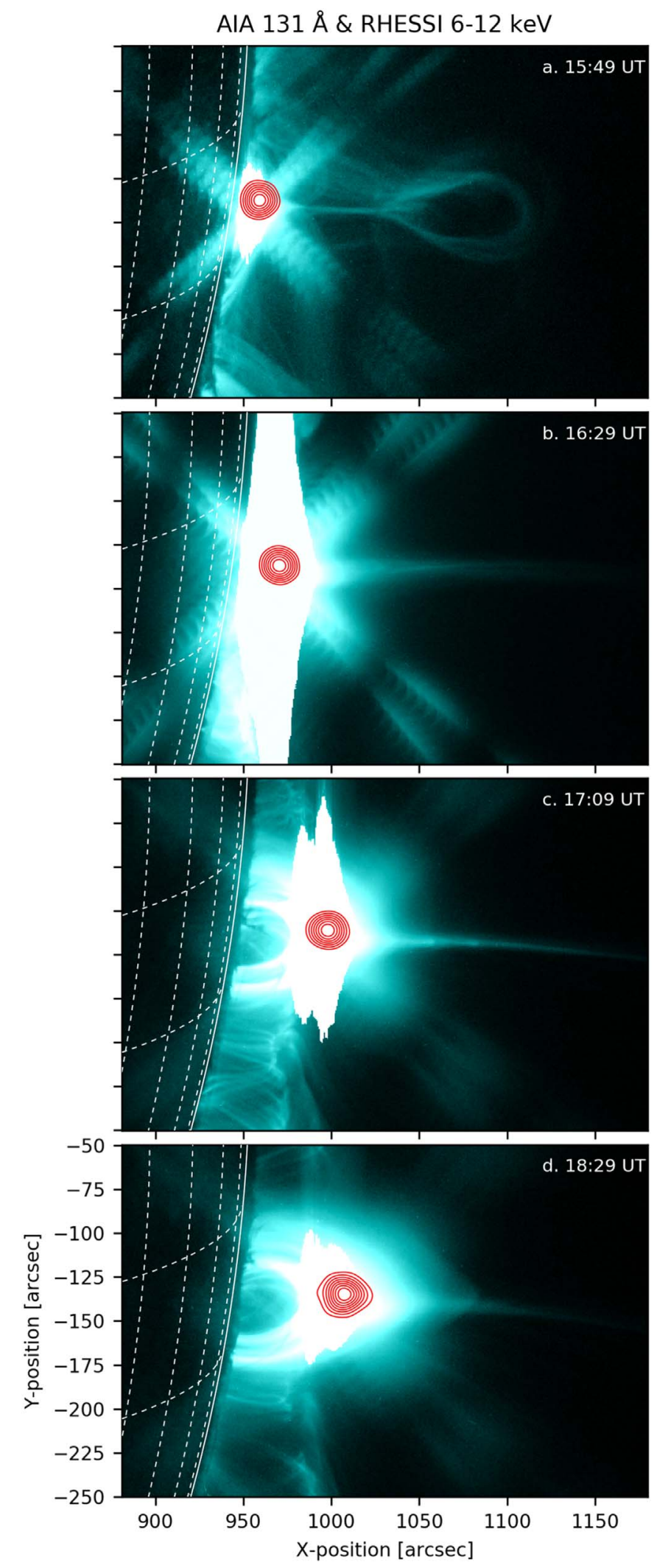

Figure 4. RHESSI contours of the $6-12 \mathrm{keV}$ source overplotted on $131 \AA$ images at four stages of the event. As in Figure 1, the pre-eruption plasmoid is seen in (a). Following the eruption, the evolution of the EUV arcade is accompanied by the ascension of the soft X-ray source to higher altitudes (c)-(d). The 12-25 keV source is not plotted here as it shows similar evolution to the $6-12 \mathrm{keV}$, but both are shown in Figure 5. energized and thus hotter, whereas lower loops are cooling into the $6-12 \mathrm{keV}$ bandpass. It should be noted that the $12-25 \mathrm{keV}$ source may include some nonthermal emission together with the thermal soft X-ray emission.

The soft X-ray source rises throughout the flare, evolving rapidly during the impulsive phase with a velocity of $17 \mathrm{~km} \mathrm{~s}^{-1}$, slowing down to $\sim 5 \mathrm{~km} \mathrm{~s}^{-1}$ just after the impulsive phase, and then to $2 \mathrm{~km} \mathrm{~s}^{-1}$ later in the decay phase. This motion is similar to that reported by Gallagher et al. (2002). However, here the impulsive phase velocity of $17 \mathrm{~km} \mathrm{~s}^{-1}$ is faster, but the decay phase linear speed is comparable. The higher velocity during the impulsive phase is presumably related to the extremely fast $\left(\sim 3000 \mathrm{~km} \mathrm{~s}^{-1}\right)$ associated coronal mass ejection (Gopalswamy et al. 2018; Morosan et al. 2019).

It is interesting to note that the bursty, larger amplitude, QPP associated with the impulsive phase occur during a time when the soft X-ray source is rapidly rising. The decay phase pulsations, on the other hand, occur when this evolution slows down, and perhaps may explain the more stable, lower amplitude nature of the pulsations observed during this time.

\subsection{AIA Loop Contractions and Downflows}

In order to enhance the features observed in the AIA $131 \AA$ images, a sequence of running difference images was produced. This allows us to investigate and track loop features and motions throughout the flare and relate them to the QPP. The running difference animation (see Figure 6) shows the plasmoid eruption with the flare emission at lower altitudes. In the early stages of the flare and during the impulsive phase, the saturated pixels dominate the running difference images and it is difficult to distinguish any features of interest. Later in the event, during the decay phase, there is clear evidence of downward retracting loops/blobs that follow a sunward direction along the current sheet that hit the top of the newly formed cusp-shaped loops. Not only is material moving downward toward the cusp-top of the flare, but also material is observed to be moving down the legs of the loops below the saturated pixels in the flare arcade.

Figures 6(a)-(c) and (d)-(f) display three consecutive running difference images at two separate times to demonstrate motions observed in the animation and described above. In Figures 6(a)-(c) we highlight the current sheet and downward directed motions presumably from the reconnection site. The red oval and arrow in (a) point to a feature that is observed to move down toward the arcade structure (b) and then form cuspshaped loops (c). This downward moving feature is likely to be associated with supra-arcade downflows (Savage \& McKenzie 2011) originating from newly reconnected field lines higher up along the bright linear feature that propagate toward the flare arcade apex. In Figures 6(d)-(f), the downward motions in the loop legs are shown below the saturated pixels. The red circles and arrows point to the bright feature of interest. The brightening is observed to propagate down the legs of the loop, beginning at the top (d) just below the saturated region and then travel down along the southern leg of the loop toward the southern footpoint. These motions in the loop leg are found to have a $\sim 80 \mathrm{~s}$ travel time from the loop top to the footpoint.

To track the downward contracting loop motion observed in Figures 6(a)-(c), a simulated slit is placed along the current sheet, noted by the white dashed line in Figure 6(c). The slit is $10^{\prime \prime}$ wide 


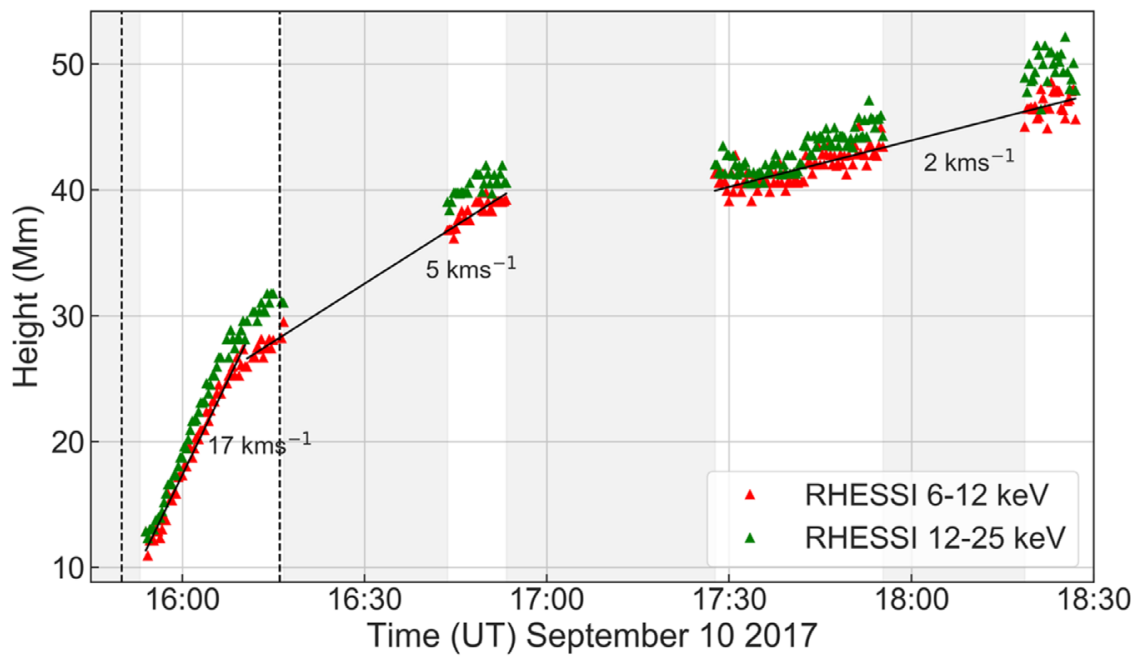

Figure 5. Projected height (in the plane of the sky) of the centroids of the 6-12 keV and 12-25 keV sources shown in red and green, respectively, plotted as a function of time. The velocities were determined by the fits shown by the black lines. The vertical dashed lines indicate the impulsive phase, as in Figure 1. Gaps in the data indicate RHESSI night (shaded regions) when it is unavailable to make observations.
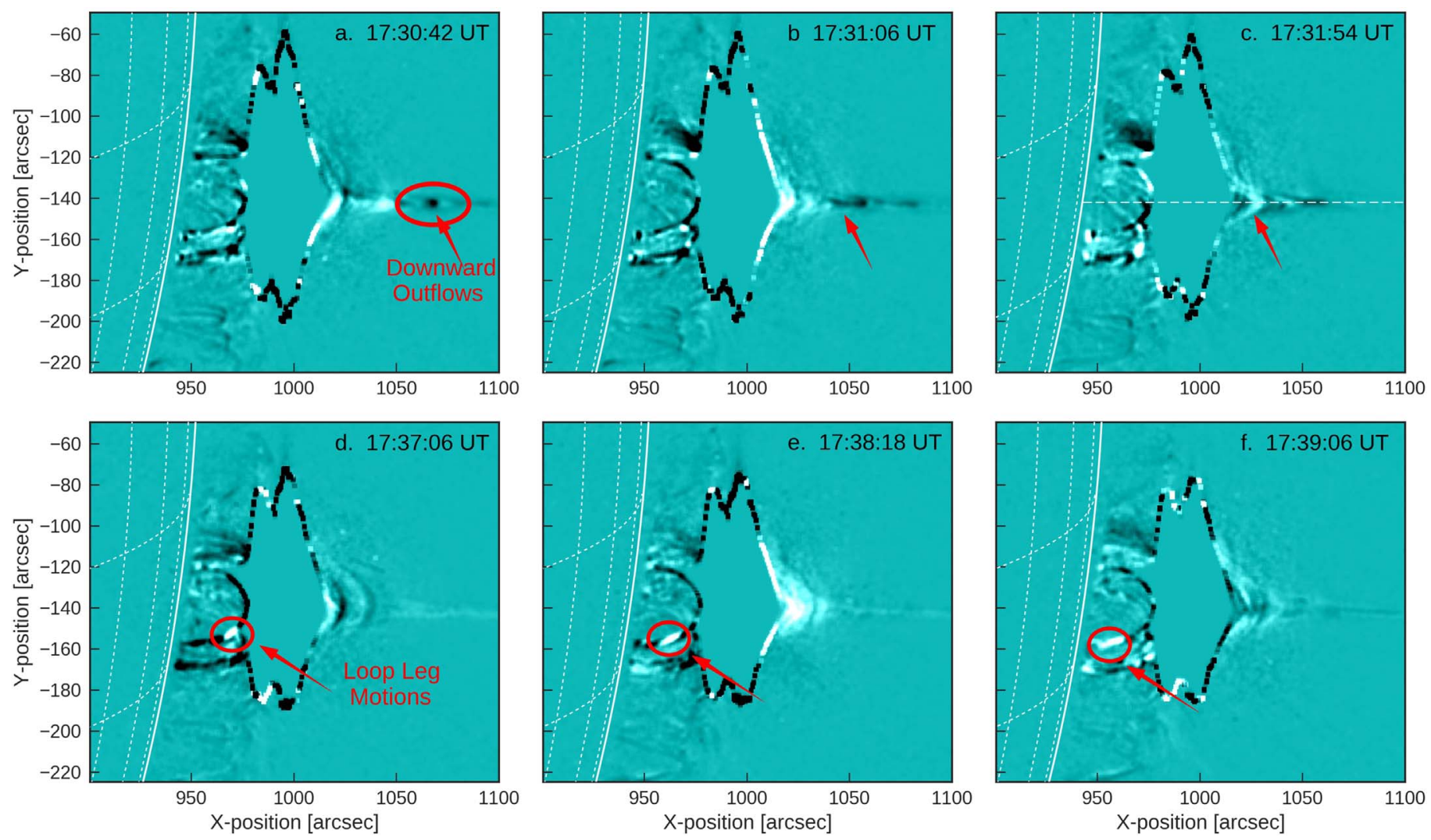

Figure 6. Snapshots from an animation of the AIA 131 A running difference images. (a)-(c) show consecutive times to highlight the downward contracting loops/ plasmoids that travel along the current sheet at impact the underlying arcade to form new post-flare loops. (d)-(f) show the other interesting feature noted in the animation. Loop leg motions are seen throughout the decay phase, once the saturation in the AIA pixels have subsided. The animation includes a synchronized time series of the AIA $131 \AA$ and GOES 1-8 $\AA$ detrended fluxes. The animation runs from 15:50 to 19:00 UT on 2017-09-10.

(An animation of this figure is available.)

and pixels are averaged over this slit to produce the space-time plot in Figure 7. Each moving feature is observed as a dark intensity track, which we have identified by eye and fit with the black dashed markings. At the beginning of the event, the plasmoid eruption is clearly seen. Similar to Liu et al. (2013), we fit each feature with a simple expression for the projected height as a function of time. The eruption is observed to have an initial velocity of $120 \mathrm{~km} \mathrm{~s}^{-1}$ and an acceleration of approximately $2 \mathrm{~km} \mathrm{~s}^{-2}$. These values are consistent with the CME velocity at this time following the eruption reported by Morosan et al. (2019).

The large gap between $\sim 950$ and 1000 arcsec in Figure 7 tracks the area of saturated pixels, and this makes it difficult to 
determine the loop top in AIA $131 \AA$ images. The saturated pixels, however, are clearly seen to rise in altitude during the impulsive phase followed by a more stable increase in the decay phase, similar to the RHESSI source heights in Figure 5. The downward contracting loops above the cusp shaped flare loops are identified as black dashed tracks in the space-time plot (Figure 7). The velocities of these downward contracting loops are in the range of $\sim 100-200 \mathrm{~km} \mathrm{~s}^{-1}$ at higher altitudes, followed by a deceleration once they arrive at the flare cusp top, where an average velocity of approximately $5-20 \mathrm{~km} \mathrm{~s}^{-1}$ is found. These values are consistent to those discussed in Savage \& McKenzie (2011) and Liu et al. (2013).

The detrended AIA $131 \AA$ light curve from Figure 1(h) is plotted above the space-time plot to compare the observed timings of the QPP to that of the observed EUV motions along the current sheet. The decay phase portion of the light curve is multiplied by a factor of 3 to aid with the visual comparison, marked in red. During the impulsive phase (from 15:50 UT to approximately 16:15 UT), large amplitude QPP are observed at the same time as the rapid rise of the emitting source as new flaring loops are formed following reconnection. After this time, the altitude increase slows down and downward contracting loops/plasmoids are observed. These are clearly also observed in the animation of Figure 6. The motion of the soft X-ray source to higher altitudes in Figure 5 suggests that the reconnection site moves higher into the corona along the current sheet and continues to release energy. The sunward moving blobs are presumably a result of this continued magnetic reconnection along the current sheet. These downward-moving features reach the top of the flaring arcade and form new loops. Although challenging to quantitatively determine, it is suggestive that the QPP are coexistent with the features that propagate toward the top of the loops. The timing indicates that the QPP occur cotemporally with features that are first identified at higher altitudes along the current sheet, before they reach the flare loop top. Two example of this are highlighted by the black arrows in Figure 7.

\section{Discussion}

We have identified extensive QPP in the thermal emissions from the X8.2 flare from 2017 September 10. The pulsations persist for up to three hours after the peak of the flare, providing one of the best examples of long duration QPP observed in a flaring event. The analyzed light curves demonstrate two regimes of QPP signatures; bursty larger amplitude pulsations in the impulsive phase with a period of $65 \mathrm{~s}$, and decay phase lower amplitude pulsations that extend late after the impulsive phase has ended, with a longer characteristic timescale of 150-160 s. During a solar flare, the impulsive and decay phases are dominated by physically different processes, and it is likely that two distinct mechanisms are underpinning the production of QPP in the different phases.

The impulsive phase of the solar flare is dominated by explosive energy release and particle acceleration due to the rapid reconfiguration of the magnetic field and the plasmoid/ CME eruption. The prompt rise of the soft X-ray source during this time suggests that the reconnection site moves quickly $\left(\sim 17 \mathrm{~km} \mathrm{~s}^{-1}\right)$ to higher altitudes along the current sheet. In this way, it is difficult to interpret the impulsive phase QPP in terms of standing MHD wave modes as it is unlikely that they could be supported in the flaring loops during this complex evolution. It is more likely that the QPP at this time are related to the dominant processes of energy release and particle acceleration that have some associated characteristic timescale.

For example, the recent work of Thurgood et al. (2017) has shown that reconnection at a 3D null point can proceed in a timedependent periodic fashion and also periodically excite propagating MHD waves. This inherent property of oscillatory reconnection may play a role in producing the observed QPP. Other works have suggested that QPP are related to the formation and dynamics of plasmoids in flare current sheets (e.g., Kliem et al. 2000). Moreover, Guidoni et al. (2016) built on the work of Drake et al. (2006) and investigated sunward moving plasmoids formed during reconnection in the simulation of an eruptive flare. They demonstrated that magnetic islands could trap and accelerate electrons that then interact with the flare arcade to produce emission. The discrete acceleration episodes associated with the generation and interaction of magnetic islands in a flaring current sheet could result in the observed pulsations in emission. The accelerated electrons could then precipitate to the chromosphere leading to the observed QPP in the hot soft X-ray emitting plasma (similar to the Neupert effect; Neupert 1968). We did not study the hard X-ray light curves for this flare given the fact that they were nonuniform given the gaps in the data (due to RHESSI nighttime and long duration nature of the flare) making it difficult to perform the same robust periodicity analysis. Pulsations in hard X-ray are not directly evident by eye, which can be the case of many QPP events (e.g., Hayes et al. 2016). However, this may be due to the fact that the flare was partially occulted (Gary et al. 2018).

Another possible scenario is that the QPP are a manifestation of reconnection jets (the fast downflows associated with magnetic reconnection) that interact with the ambient plasma above the loop top and excite local oscillations. Takasao \& Shibata (2016) performed a set of 2D MHD simulations of a solar flare and studied these oscillations excited by the reconnection outflows. They found that the above-the-loop-top region was full of shocks and oscillations and the region generated quasi-periodic propagating fast magnetoacoustic waves (QPFs). The oscillations were controlled by multiple shocks in the region produced as a result of the collision of the reconnection outflows with the reconnected flaring loops piled up below. Their study also found quasiperiodic oscillations of the termination shock strength. It has been noted that termination shocks could be a possible site for particle acceleration (e.g., Chen et al. 2015), and hence a quasi-periodic oscillation of the termination shock at the top of the flare loop could accelerate nonthermal electrons quasi-periodically, causing QPP in the soft X-ray flux also. More recently Takahashi et al. (2017) performed 2D simulations of magnetic reconnection that occurs below an erupting $\mathrm{CME}$, and similarly found oscillations in the above-the-loop-top region, even in the case of plasmoiddriven reconnection. In this scenario, it was found during a solar eruptive event that the impulsive phase QPP occur when the $\mathrm{CME}$ acceleration is peaking, which is the case for this flare.

The model of collapsing magnetic traps within the flaring loop-top region may also be a possible mechanism to explain the X-ray QPPs (see Jakimiec \& Tomczak 2010, 2012). In this model, reconnected at the top of a cusp-shaped structure generates a sequence of magnetic traps that are then compressed following collision with the stronger magnetic field below. The compression results in particle acceleration, and increases the gas and magnetic pressure inside the trap. Once this pressure increases, the traps then expand and soft X-ray emitting plasma can then fill the trap. Such a compression and expansion sets up 


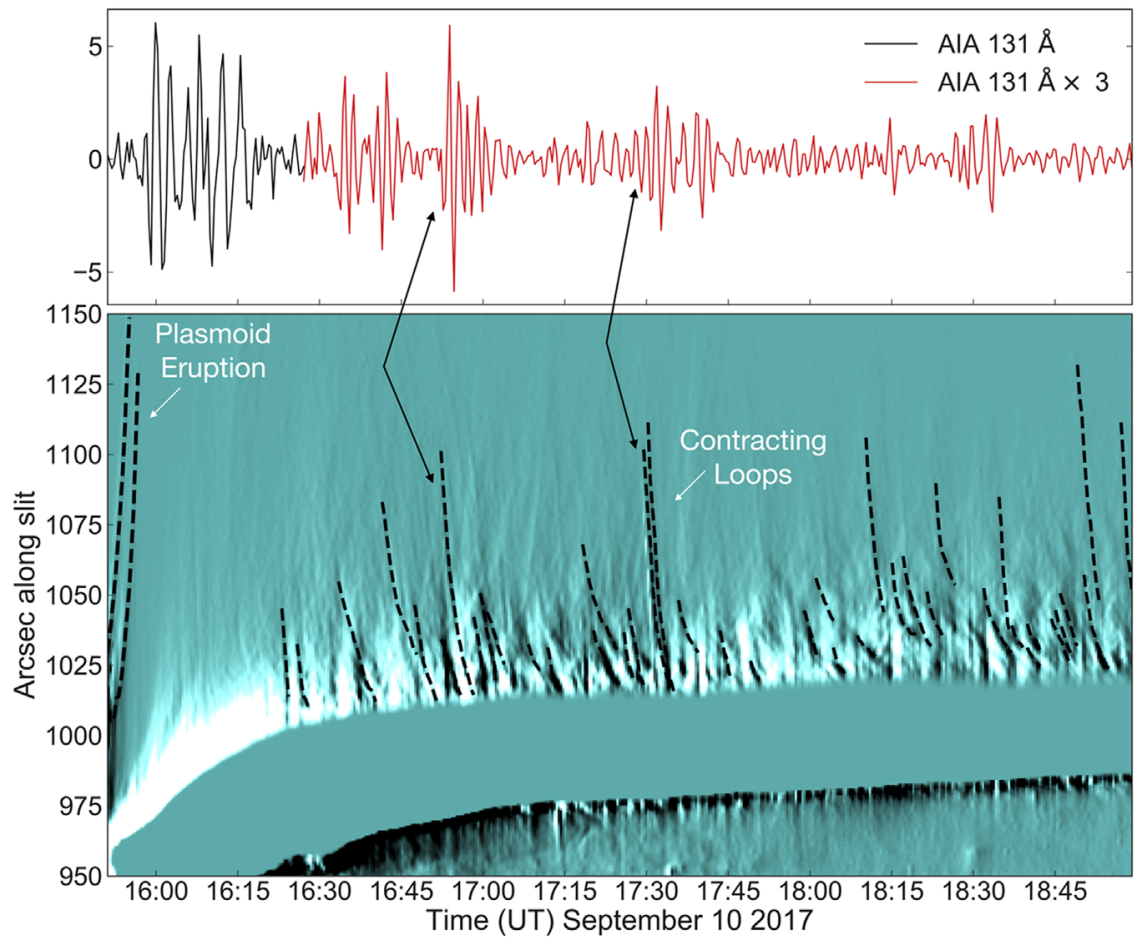

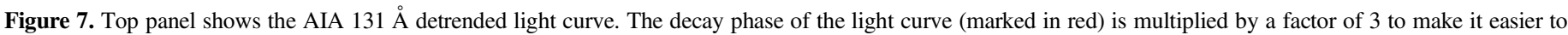

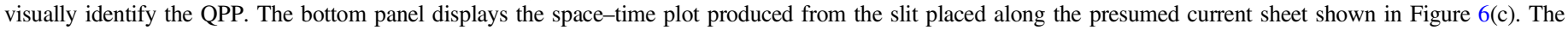

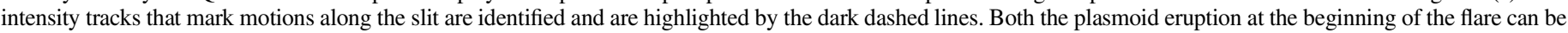

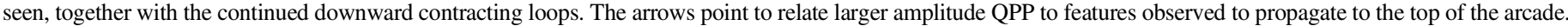

a magnetoacoustic oscillation of the trap. The periods of the periodicity in the decay phase could be interpreted as due to changes of the length scales of the oscillating traps later in the flare evolution (Jakimiec \& Tomczak 2012).

Unlike the impulsive phase, when reconnection processes and magnetic configuration evolution dominate, the decay phase is characterized by a more stable configuration of post-flare loops. In this way, it is possible that the QPP at this time are a manifestation of MHD wave modes supported in the post-flare loops. The AIA running difference animation (see Figure 6) shows extended downward contractions of plasmoids that reach the top of the post-flare arcade and presumably form new loops. The downward contractions could excite MHD wave oscillations in the underlying arcade, resulting in density perturbations of the post-flare loops. The density perturbations would then be observed in the hottest thermal plasma of the newly formed loops and hence are detectable in the soft X-ray and $131 \AA$ wavebands.

Recent numerical simulations by Jelínek et al. (2017) support this idea, as their simulation of magnetic reconnection in a current sheet found that plasmoids formed by the tearing mode instability could interact with the underlying flaring arcade and generate standing transverse MHD waves of the slow magnetoacoustic type. Slow magnetoacoustic waves can perturb the plasma density of a flaring loop and produce the observed modulation in the thermal emissions (e.g., Wang 2011). Moreover, it has been shown that an energy deposition at a flaring loop apex preferably excites the second spatial harmonic of the mode in a hot flaring loop (Nakariakov et al. 2004; Tsiklauri et al. 2004; Selwa et al. 2005), with periods in the range of 10-300 s. The period observed in this flare ( $\sim 150-160 \mathrm{~s}$ during the decay phase) lies within this range and hence slow magnetoacoustic waves may be a reasonable explanation. The period, $P$ (in s), of the second harmonic of the slow magnetoacoustic wave is given by $P=6.7 L / \sqrt{T}$, where $L$ is the loop length in $\mathrm{Mm}$, and $T$ is the average temperature in the loop (in MK). The loop length can be readily estimated if we assume that the loops have a semicircular shape, $L=\pi h$, where $h$ is the soft X-ray source altitude that can be determined from Figure 5. During the decay phase the loop lengths are in the range of $125-150 \mathrm{Mm}$. With the observed period of $150 \mathrm{~s}$, the assumption of a slow magnetoacoustic wave suggests a plasma temperature of $35-40 \mathrm{~K}$. This temperature estimate is much higher than what is found from GOES temperature of $\sim 15 \mathrm{MK}$, shown in Figure 8, during the decay phase. Hence this questions the validity of the slow magnetoacoustic interpretation.

Indeed, other MHD wave modes can perturb the flaring plasma. The global sausage mode is often discussed in terms of thermal QPP, given that it is a compressive wave mode and can cause density variations within the loop to produce observed soft X-ray and EUV modulation (Van Doorsselaere et al. 2011; Tian et al. 2016). However, the sausage mode has a wavenumber cutoff, and cannot support long period oscillations (Nakariakov et al. 2012). The period here of $\sim 150 \mathrm{~s}$ is too long to be interpreted as sausage mode oscillations of the flaring loops.

The kink mode can also cause density perturbations when it oscillates in the vertical polarization-i.e., up and down in the same direction of the plane of the loop rather than in the transverse horizontal motion often observed (Aschwanden \& Schrijver 2011). In the vertical polarization, the loop length can vary, moving up and down during the oscillations. This stretching and shrinking of the loop is likely to cause a density modulation of the central loop cross section, hence producing a sausage-like cross sectional and density oscillation in the loop (Aschwanden \& Schrijver 2011). For the kink-mode to move in this way, an initial excitation in the plane of the loop is 


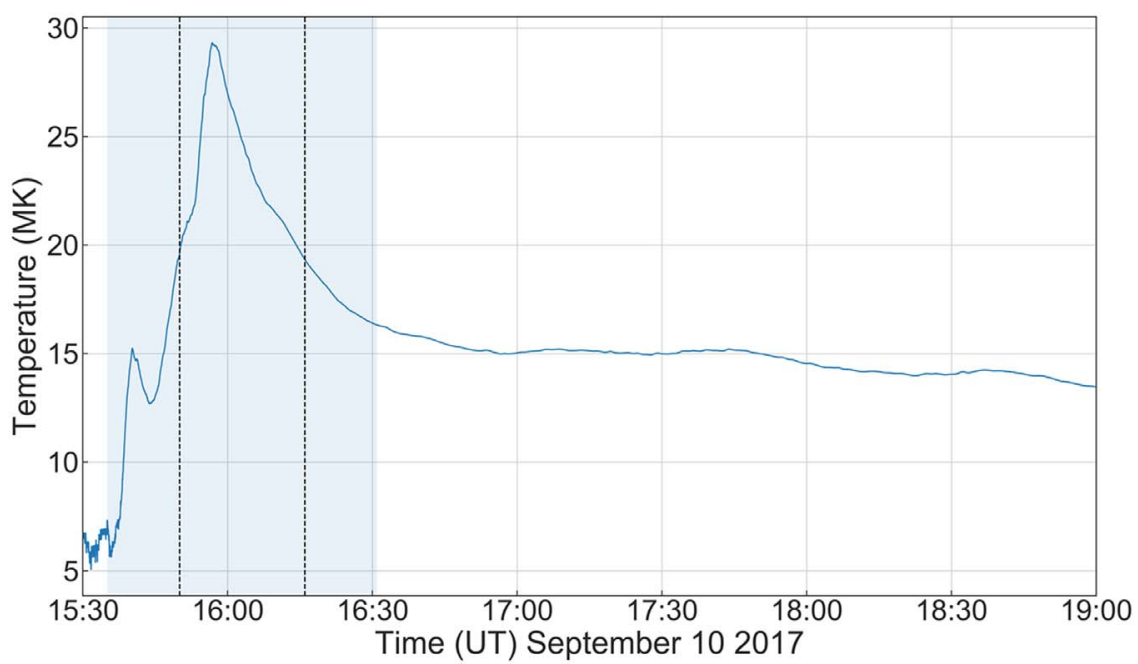

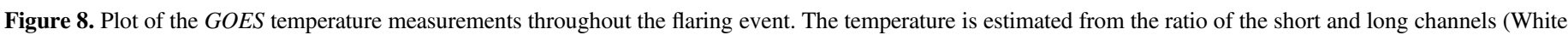

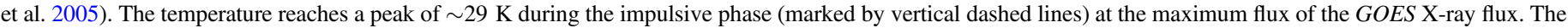

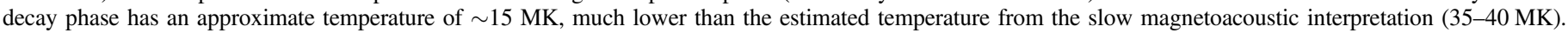

required. For this event, the downward contracting loops at the top of the flare cusp could be a viable exciter. The expected changes of the soft X-ray emission in this case are expected to be small, a fraction of the overall emission, which is consistent with the observations. This interpretation has been used in the interpretation of soft X-ray pulsations for another decay phase QPP event (Dennis et al. 2017). The period of the kink mode is given by $P=2 L / C_{k}$, where here $L$ is the loop length, and $C_{k}$ is the phase speed of the kink mode. The phase speed of the kink mode in coronal flaring loops can be approximated to be $\sqrt{2} V_{A}$, where $V_{A}$ is the internal Alfvén speed of the loop (Aschwanden 2005). Using the period values of $150 \mathrm{~s}$, and a loop length of 125-150 Mm (Figure 5), an interpretation of the QPP in terms of the kink mode oscillations suggests an Alfvén speed of $\sim 1178-1414 \mathrm{~km} \mathrm{~s}^{-1}$. These are reasonable velocities for coronal loops (e.g., Aschwanden 2005), and perhaps suggests that the prolonged soft X-rays QPP are a manifestation of vertical kink mode oscillations in the post-flare loops.

MHD wave modes identified in coronal loops often display distinct damped oscillatory patterns (White \& Verwichte 2012). Similarly, solar flare QPP have been reported to demonstrate damped signatures in the decay phase (e.g., Hayes et al. 2016). The observations of persistent QPP identified in the decay phase of this flare suggest that there is a continued renewal or excitation of MHD modes in the flaring arcade. The continued downward contractions observed in AIA $131 \AA$ along the current sheet fit this scenario and explain why continued pulsations are observed for such a long time during this flare. It should also be noted that MHD wave mode oscillations could be present during the impulsive phase. In this case, however, it could be expected that the overall energy release and particle acceleration dominates and their specific oscillations cannot be identified.

\section{Conclusions}

Long duration QPP have been investigated in the large X8.2 solar flare on 2017 September 10. Soft X-ray thermal QPP are observed throughout the impulsive phase and late into the decay phase of the flare. The decay phase pulsations are of particular interest, persisting up to $3 \mathrm{hr}$ after the peak in the GOES X-ray flux. Similar to other reports of soft X-ray QPP, an evolution of the characteristic timescale of the pulsations is observed, from shorter periods in the impulsive phase $(\sim 65 \mathrm{~s})$ to longer periods $(\sim 150 \mathrm{~s})$ in the decay phase (Simões et al. 2015; Hayes et al. 2016; Dennis et al. 2017). These observations may reflect that this evolution is an intrinsic feature of flaring QPP, perhaps attributed to different dominant drivers of the pulsations in different stages of the flare, or instead that the timescale is related to the evolution of the loop length scales that is observed to increase throughout a flaring event.

A key finding in this work is that the decay phase pulsations coexist with observations of extended downward motions seen in the AIA $131 \AA$ images along the current sheet that impact the top of the flaring arcade. The coexistent QPP and observed downward contractions suggest that the dynamical structure of the current sheet formation and the associated CME eruption play an important role in the generation of decay phase QPP signatures. Furthermore, this study may help explain why no correlations were found between detected QPP periods and global active region properties such as active region size, average magnetic field strength, or dipole separation in the recent statistical study performed by Pugh et al. (2017). The results here instead suggest that QPP signatures are most likely related to the loop length scales of the flare and the magnetic configuration. Future work is now required to perform a similar statistical study investigating the relationship between QPP signatures, throughout both the impulsive and decay phases, to the loop length scales and between both compact and eruptive flares.

This work has been supported by an Enterprise Partnership Scheme studentship from the Irish Research Council (IRC) between Trinity College Dublin and ADNET Systems Inc. D.E.M. acknowledges the Finnish Centre of Excellence in Research of Sustainable Space (Academy of Finland grant No. 1312390). We would like to thank the anonymous reviewer for the helpful feedback and comments, which improved this manuscript.

Facilities: GOES/XRS, RHESSI, SDO (EVE, AIA).

Software: SunPy (SunPy Community et al. 2015).

\section{Appendix}




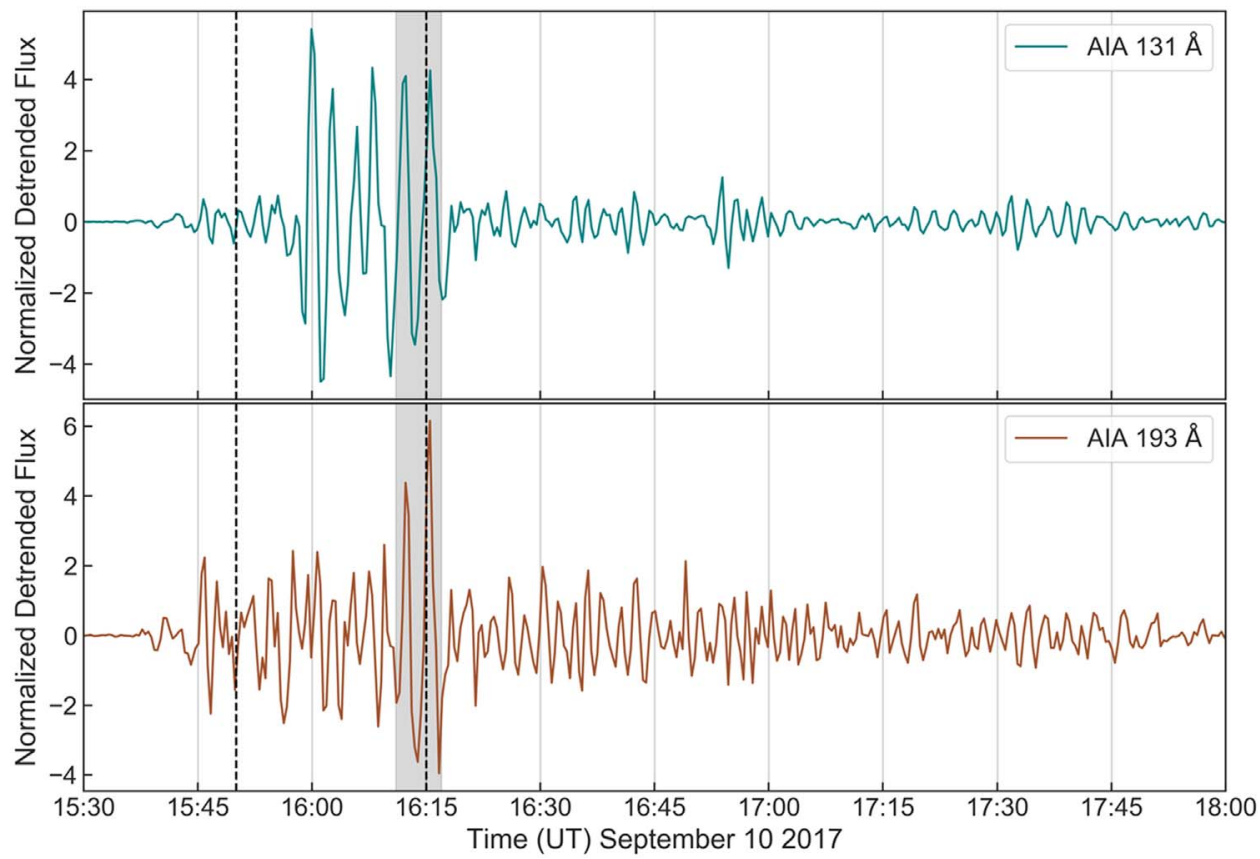

Figure 9. Detrended AIA 131 and $193 \AA$ light curves. The top panel shows the same as Figure 1(h), and the bottom panel shows the detrended light curve of the AIA $193 \AA$ A channel. The vertical dashed lines denote the impulsive phase as in Figure 1. The gray shaded region highlights the extra two pulsations that are observed in both EUV channels but not in GOES or ESP. They are shown to demonstrate that these pulsations are real as they are observed in both channels.

\section{ORCID iDs}

Laura A. Hayes (ib https://orcid.org/0000-0002-6835-2390

Brian R. Dennis (i) https://orcid.org/0000-0001-8585-2349

Jack Ireland (10 https://orcid.org/0000-0002-2019-8881

Andrew Inglis (i) https://orcid.org/0000-0003-0656-2437

\section{References}

Anfinogentov, S., Nisticó, G., \& Nakariakov, V. M. 2013, A\&A, 560, A107 Anfinogentov, S. A., Nakariakov, V. M., \& Nisticó, G. 2015, A\&A, 583, A136 Aschwanden, M. J. 2005, Physics of the Solar Corona. An Introduction with Problems and Solutions (2nd ed.; New York: Springer)

Aschwanden, M. J., \& Schrijver, C. J. 2011, ApJ, 736, 102

Brosius, J. W., Daw, A. N., \& Inglis, A. R. 2016, ApJ, 830, 101

Chen, B., Bastian, T. S., Shen, C., et al. 2015, Sci, 350, 1238

Cheng, X., Li, Y., Wan, L. F., et al. 2018, ApJ, 866, 64

De Moortel, I., \& Nakariakov, V. M. 2012, RSPTA, 370, 3193

Dennis, B. R., Tolbert, A. K., Inglis, A., et al. 2017, ApJ, 836, 84

Dolla, L., Marqué, C., Seaton, D. B., et al. 2012, ApJL, 749, L16

Dominique, M., Zhukov, A. N., Dolla, L., et al. 2018, SoPh, 293, 61

Drake, J. F., Swisdak, M., Che, H., et al. 2006, Natur, 443, 553

Fleishman, G. D., Bastian, T. S., \& Gary, D. E. 2008, ApJ, 684, 1433

Gallagher, P. T., Dennis, B. R., Krucker, S., et al. 2002, SoPh, 210, 341

Gary, D. E., Chen, B., Dennis, B. R., et al. 2018, ApJ, 863, 83

Gopalswamy, N., Yashiro, S., Mäkelä, P., et al. 2018, ApJL, 863, L39

Gruber, D., Lachowicz, P., Bissaldi, E., et al. 2011, A\&A, 533, A61

Guidoni, S. E., DeVore, C. R., Karpen, J. T., et al. 2016, ApJ, 820, 60

Hayes, L. A., Gallagher, P. T., Dennis, B. R., et al. 2016, ApJL, 827, L30

Huang, J., Kontar, E. P., Nakariakov, V. M., et al. 2016, ApJ, 831, 119

Hurford, G. J., Schmahl, E. J., Schwartz, R. A., et al. 2002, SoPh, 210, 61

Inglis, A. R., \& Dennis, B. R. 2012, ApJ, 748, 139

Inglis, A. R., Ireland, J., Dennis, B. R., et al. 2016, ApJ, 833, 284

Inglis, A. R., Ireland, J., \& Dominique, M. 2015, ApJ, 798, 108

Jakimiec, J., \& Tomczak, M. 2010, SoPh, 261, 233

Jakimiec, J., \& Tomczak, M. 2012, SoPh, 278, 393

Jelínek, P., Karlický, M., Van Doorsselaere, T., et al. 2017, ApJ, 847, 98

Kass, R., \& Raftery, A. 1995, J. Am. Stat. Assoc., 90, 773

Kliem, B., Karlický, M., \& Benz, A. O. 2000, A\&A, 360, 715

Kolotkov, D. Y., Pugh, C. E., Broomhall, A.-M., et al. 2018, ApJL, 858, L3

Kupriyanova, E. G., Kashapova, L. K., Reid, H. A. S., et al. 2016, SoPh, 291,3427
Lemen, J. R., Title, A. M., Akin, D. J., et al. 2012, SoPh, 275, 17

Li, D., Ning, Z. J., \& Zhang, Q. M. 2015, ApJ, 807, 72

Li, Y., Xue, J. C., Ding, M. D., et al. 2018, ApJL, 853, L15

Lin, R. P., Dennis, B. R., Hurford, G. J., et al. 2002, SoPh, 210, 3

Liu, W., Chen, Q., \& Petrosian, V. 2013, ApJ, 767, 168

Liu, W., Jin, M., Downs, C., et al. 2018, ApJL, 864, L24

McLaughlin, J. A., Nakariakov, V. M., Dominique, M., et al. 2018, SSRv, 214,45

McLaughlin, J. A., Verth, G., Fedun, V., et al. 2012, ApJ, 749, 30

Milligan, R. O., Fleck, B., Ireland, J., et al. 2017, ApJL, 848, L8

Morosan, D. A., Carley, E., Hayes, L. A., et al. 2019, NatAs, tmp, 210

Murray, M. J., van Driel-Gesztelyi, L., \& Baker, D. 2009, A\&A, 494, 329

Nakariakov, V. M., Foullon, C., Myagkova, I. N., et al. 2010, ApJL, 708, L47

Nakariakov, V. M., Foullon, C., Verwichte, E., et al. 2006, A\&A, 452, 343

Nakariakov, V. M., Hornsey, C., \& Melnikov, V. F. 2012, ApJ, 761, 134

Nakariakov, V. M., \& Melnikov, V. F. 2009, SSRv, 149, 119

Nakariakov, V. M., Tsiklauri, D., Kelly, A., et al. 2004, A\&A, 414, L25

Nakariakov, V. M., \& Zimovets, I. V. 2011, ApJL, 730, L27

Neupert, W. M. 1968, ApJL, 153, L59

Omodei, N., Pesce-Rollins, M., Longo, F., et al. 2018, ApJL, 865, L7

Parks, G. K., \& Winckler, J. R. 1969, ApJL, 155, L117

Press, W. H., Flannery, B. P., \& Teukolsky, S. A. 1986, Numerical Recipes in C, Vol. 2 (Cambridge: Cambridge Univ. Press)

Pugh, C. E., Nakariakov, V. M., Broomhall, A.-M., et al. 2017, A\&A, 608, A101

Savage, S. L., \& McKenzie, D. E. 2011, ApJ, 730, 98

Savitzky, A., \& Golay, M. J. E. 1964, AnaCh, 36, 1627

Schwarz, G. 1978, AnSta, 6, 461

Selwa, M., Murawski, K., \& Solanki, S. K. 2005, A\&A, 436, 701

Simões, P. J. A., Hudson, H. S., \& Fletcher, L. 2015, SoPh, 290, 3625

SunPy Community, Mumford, S. J., Christe, S., et al. 2015, CS\&D, 8, 14009

Takahashi, T., Qiu, J., \& Shibata, K. 2017, ApJ, 848, 102

Takasao, S., \& Shibata, K. 2016, ApJ, 823, 150

Thurgood, J. O., Pontin, D. I., \& McLaughlin, J. A. 2017, ApJ, 844, 2

Tian, H., Young, P. R., Reeves, K. K., et al. 2016, ApJL, 823, L16

Tsiklauri, D., Nakariakov, V. M., Arber, T. D., \& Aschwanden, M. J. 2004, A\&A, 422, 351

Van Doorsselaere, T., De Groof, A., Zender, J., et al. 2011, ApJ, 740, 90

Van Doorsselaere, T., Kupriyanova, E. G., \& Yuan, D. 2016, SoPh, 291, 3143

Wang, T. 2011, SSRv, 158, 397

Warren, H. P., Brooks, D. H., Ugarte-Urra, I., et al. 2018, ApJ, 854, 122

White, R. S., \& Verwichte, E. 2012, A\&A, 537, A49

White, S. M., Thomas, R. J., \& Schwartz, R. A. 2005, SoPh, 227, 231

Woods, T. N., Eparvier, F. G., Hock, R., et al. 2012, SoPh, 275, 115 